\title{
ABORDAJES E IMPLICACIONES EN LA CONSTRUCCIÓN DE LA IDENTIDAD PROFESIONAL DEL PSICólogo
}

\author{
Leticia Nayeli Ramírez Ramírez \\ Rosa del Carmen Flores Macías \\ Marguerite Lavallée, \\ Luiza Bontempo e Silva \\ UNAM-FACULTAD DE PSICOLOGÍA
}

\section{RESUMEN}

MÉXICO

El objetivo de este trabajo es aportar una visión interpretativa a la revisión de la literatura actual sobre la identidad profesional del psicólogo en Iberoamérica. Se realizó un estudio cualitativointerpretativo a través de la identificación de indicadores contextuales y temáticos de cinco artículos obtenidos de las principales bases de datos de Iberoamérica. Se describen cuatro dimensiones identificadas en los hallazgos: 1) características de los contextos profesionales; 2) vínculo entre epistemología e identidad profesional; 3) formación profesional vinculada a la práctica, y 4) interacción psicólogo-cliente. Los resultados son discutidos en torno a dos elementos mediadores de la identidad profesional: la epistemología personal y los contextos profesionales. Se sugiere que en futuras investigaciones se realicen análisis integrales en los que se identifiquen tanto las creencias que los estudiantes construyen sobre el conocimiento, como las características de los contextos en los que tiene lugar la práctica profesional de los estudiantes.

Palabras Clave: Identidad profesional, epistemología personal, contexto profesional, psicólogos.

\section{APPROACHES AND IMPLICATIONS IN THE CONSTRUCTION OF PROFESSIONAL IDENTITY IN PSYCHOLOGIST}

\begin{abstract}
The purpose of this work is to provide an interpretative vision of the current literature about professional identity of the psychologist in Ibero-American context. A qualitative-interpretative study was realized through the identification of contextual and thematic indicators of five articles obtained of the principal databases of Ibero-American. Four dimensions were identified on the findings: 1) characteristics of the professional contexts; 2) link between epistemology and professional identity; 3 ) professional training linked to practice; and 4) interaction psychologistclient. The results are discussed around two intermediary elements of the professional identity; the personal epistemology and the professional contexts. The comprehensive analysis is suggested for further studies, which include, the beliefs that students construct around the knowledge as well as the characteristics of the context where students professional practice is performed.
\end{abstract}

Keywords: Professional identity, personal epistemology, professional context, psychologists. 


\section{BITÁCORA DEL ARTÍ́CULO}

\section{ABORDAJES E IMPLICACIONES EN LA CONSTRUCCIÓN DE LA IDENTIDAD PROFESIONAL DEL PSICÓLOGO Leticia Nayeli Ramírez Ramírez, Rosa del Carmen Flores Macías, Marguerite Lavallée y Luiza Bontempo e Silva}

\section{Bitácora del Artículo:}

Recibido: 27 de septiembre de 2014

Aceptado: 3 de diciembre de 2014

Publicado en línea: 7 de febrero de 2015

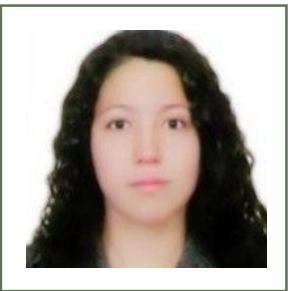

Doctorante en el programa de Psicología Educativa y del Desarrollo de la Universidad Nacional Autónoma de México (U.N.A.M.) Licenciada en Psicología por la Facultad de Estudios Superiores Iztacala (F.E.S.I.)

Ver más...

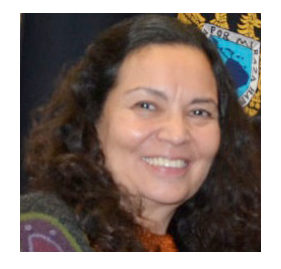

Rosa del Carmen Flores Macías UNAM - Facultad de Psicología

Correo: rcfm@unam.mx

Doctorado en Educación, Universidad Autónoma de Aguascalientes. Maestría en Psicología, Universidad Nacional Autónoma de México. Tutora del programa de Maestría y Doctorado en Psicología de la UNAM.

Ver más...

\section{Contribución de las autoras}

Leticia Nayeli Ramírez Ramírez y Rosa del Carmen Flores Macías concibieron el estudio y participaron en todos los rubros del artículo | Marguerite Lavallée y Luiza Bontempo e Silva participaron enriqueciendo con aportes teóricos y antecedentes del tema |

\section{Agradecimientos}

Se agradece el apoyo brindado por la Université Laval y la Dra. Marguerite Lavallée para la elaboración de la presente investigación.

\section{Datos de filiación de las autoras}

Rosa del Carmen Flores Macías | División de Posgrado, Facultad de Psicología CU, UNAM | Leticia Nayeli Ramírez Ramírez | FES Iztacala | 


\section{TABLA DE CONTENIDO}

INTRODUCCIÓN

Abordajes en torno al concepto de identidad profesional, 153

Modelos sobre desarrollo de la identidad profesional del psicólogo, 154

Construcción de la identidad del psicólogo, 155

MÉTOdo

Rango de la búsqueda, 156

Unidades de análisis, 156

Núcleos temáticos, 156

Artículos encontrados, 156

Criterio de inclusión y exclusión de artículos, 157

Procedimiento, 157

RESULTADOS

Discusión

CONCLUSIONES

Implicaciones para futuras investigaciones, 161

REFERENCIAS

MetA-AnÁlisis del Artículo

Dimensión Cuantitativa, 165

Dimensión Cualitativa, 167 
Introducción

$\mathrm{E}$ $\mathrm{n}$ las últimas décadas la educación superior a nivel mundial ha experimentado una serie de transformaciones en lo tocante a su organización, reformas y paradigmas que la sustentan, desde las cuales se propone que las Instituciones de Educación Superior (IES) ajusten sus planes de estudio y currículos hacia la articulación entre el conocimiento científico y las demandas sociales, esto incluye, por lo tanto, que la formación de profesionales esté orientada hacia la promoción de componentes del saber hacer y el saber cómo, la experticia y la reflexión (UNESCO, 2009).

Bajo este contexto, el campo de estudio sobre formación profesional cobra relevancia debido a su interés por comprender los factores que intervienen para que el estudiante en formación despliegue formas de pensamiento y actuación expertas. La vasta literatura sobre formación profesional y actuación competente del psicólogo (véanse, por ejemplo: Ducheny, Alletzhauser, Crandell y Schneider, 1997; Elman, Illfelder-Kaye y Robiner, 2005; Kaslow, 2004; Rodolfa et al., 2005) ha señalado como un componente central del desarrollo profesional a la identidad profesional, al ser ésta la que posibilita que el profesional desarrolle un sentido de pertenencia a la profesión, seguridad respecto al rol profesional y la apropiación de normas y valores propios de la disciplina.

No obstante su importancia, la construcción de la identidad profesional de los psicólogos ha sido poco estudiada (Ruvalcaba-Coyaso, Uribe y Gutiérrez, 2011) en comparación con las identidades de los docentes (Beijaard, Meijer y Verloop, 2004), médicos (Goldie, 2012) y enfermeras (Johnson, Cowin, Wilson y Young, 2012; Serra, 2008).

Aunado a lo anterior, el concepto de identidad profesional entraña múltiples acepciones que complejizan su definición; en una revisión reciente de las investigaciones que exploran la identidad profesional en diversos campos disciplinarios Trede, Macklin y Bridges (2012) encontraron que existe un uso plural y ambiguo del concepto de identidad profesional, el cual es usado en diversos contextos, ya sea para enfatizar el desarrollo profesional (las habilidades y procesos involucrados en el aprendizaje profesional), 0 bien, la socialización profesional (las actitudes, valores y roles de la comunidad profesional).

Bajo este panorama heterogéneo, se delinea la importancia de realizar indagaciones en la literatura actual sobre la identidad profesional del psicólogo que permitan comprender las características de los componentes que se ponen en juego en el proceso de construcción identitario y que contribuyan a la reflexión de los abordajes teóricos y pedagógicos que coadyuven en el eventual desarrollo de estrategias orientadas a la formación de profesionales de la psicología.

Por lo que en el presente artículo pretendemos aportar una visión interpretativa (Fernández-Ríos y BuelaCasal, 2009) de la literatura actual sobre la identidad profesional del psicólogo en Iberoamérica con el fin de abonar a la comprensión de los elementos implicados en el proceso de construcción identitario.

Para abordar lo anterior, en la primera parte, se delinearán las principales aportaciones teóricoempíricas de los abordajes sobre identidad profesional a nivel internacional. En seguida, se presentará un análisis de las investigaciones recientes en el contexto de Iberoamérica sobre la identidad profesional del psicólogo, y, finalmente se discutirá sobre los elementos, identificados en la literatura, como mediadores en la construcción de la identidad profesional de los psicólogos.

\section{Abordajes en torno al concepto de identidad profesional}

Las tradiciones teóricas que son el preámbulo de los planteamientos sobre identidad profesional han sido propuestas desde dos polos, por una parte, las teorías sobre desarrollo personal correspondientes a los diversos teóricos de la psicología y, por otra, el polo estructural o colectivo abordado por las diversas teorías sociales (Dubar, 1992).

Desde las posturas sobre desarrollo de la identidad personal, Erik Erikson es uno de los pioneros en señalar que la identidad personal se desarrolla a través de ocho fases vinculadas al ciclo de la vida, en las que, al final de la evolución, se produce una crisis de identidad correspondiente al momento de encrucijada; la más notable es la que se produce en la adolescencia o más adelante en la vida adulta cuando se enfrentan dificultades particulares. Si bien las bases propuestas por Erikson abonan un progreso en el estudio de la 
identidad, en su trabajo se encuentra una visión de la identidad como un proceso que finaliza en la vida adulta, en la que la identidad alcanza un status fijo, así la identidad es vista como un proceso que se cristaliza en un producto; la identidad única, verdadera y estable (Kaufmann, 2004).

Desde una posición social, el trabajo de George Herbert Mead aporta unavisión dela identidad ${ }^{4}$ como interacción social; el Yo es esencialmente una estructura cultural y social que deviene en las interacciones con los otros. A partir de dicha tesis, autores posteriores han sugerido conceptuar a la identidad como un proceso dinámico y continuo, resultado de una negociación permanente entre la persona y su contexto social (Goffman, 1959).

Frente a ambos puntos de vista, el concepto de identidad personal se encuentra en una suerte de disyuntiva entre un entendimiento individual y otro social, que ha sido trasladada al estudio de la identidad profesional.

Las líneas de investigación que se han constituido sobre la identidad profesional del psicólogo se orientan hacia estas dos vertientes; una se enfoca a explorar el desarrollo personal y las experiencias por las que atraviesan los psicólogos en diferentes momentos de su formación profesional, y otras se orientan a indagar las características de los contextos formativos a través de los cuales los psicólogos construyen sus identidades profesionales. A continuación presentamos los principales antecedentes de ambas líneas de investigación a nivel internacional.

\section{Modelos sobre desarrollo de la identidad profesional del psicólogo}

El conjunto de estudios que integran esta línea de investigación tienen por objetivo describir el desarrollo de la identidad profesional del psicólogo en diferentes momentos de su formación, desde la licenciatura hasta el momento de cursar especializaciones en el área clínica.

El trabajo realizado por Rønnestad y Skovholt (2003) contribuye a describir el desarrollo profesional de 100 terapeutas norteamericanos con diferentes niveles de experiencia (desde estudiantes de pregrado a estudiantes de doctorado).

Los autores realizaron entrevistas semiestructuradas, a través de las cuales exploraron las percepciones que tenían los terapeutas de sus propias experiencias en el trabajo de la psicoterapia. De este estudio, derivaron un modelo de seis fases (de ayudante, comenzando a ser estudiante, del estudiante avanzado, del profesional novato, del profesional con experiencia y del profesional experto) mediante el que plantean el desarrollo profesional como un proceso de individuación.

Durante dicho proceso, el psicólogo transita de una fase de ayudante, en la que se guía por las concepciones de lo que es asistir a otros y desarrolla una fuerte identificación con el cliente, lo cual impide examinar su práctica de una manera reflexiva, hasta llegar a la fase del profesional experto, en la que el profesional tiene varios años de experiencia, y un rol altamente congruente con sus autopercepciones (sus valores, intereses, actitudes), lo que hace posible que capacite a otros no expertos.

Los resultados de la investigación establecen una recíproca relación entre el nivel de asertividad con el que los terapeutas afrontan las dificultades en la relación con el cliente y las experiencias de crecimiento profesional, lo cual involucra la movilización de procesos identitarios, desde los cuales se integran aspectos personales y profesionales para la toma de decisiones ante los dilemas que entraña la práctica profesional del terapeuta.

Por su parte, Nelson y Jackson (2003) identificaron los elementos necesarios para que el terapeuta en formación desarrolle una identidad profesional. En su estudio analizaron el desarrollo de la identidad profesional en ocho estudiantes hispanos graduados como terapeutas, por medio de una investigación cualitativa. Identificaron siete elementos, a saber: a) conocimiento, b) crecimiento personal, c) experiencia, d) relaciones, e) logros, f) costos y g) percepciones sobre la profesión.

La investigación aporta datos importantes al respecto de los recursos que apoyan el desarrollo de la identidad profesional, de los cuales, los estudiantes enfatizan las relaciones que establecen, durante su formación profesional, con los otros (pares, profesores, supervisores y familia) como cruciales para apoyar positivamente su aprendizaje y crecimiento personal.

En consonancia con lo anterior, Auxier, Hughes y Kline (2003) exploraron las experiencias de identidad profesional de terapeutas en formación a nivel maestría. Para ello, se basaron en la teoría fundamentada con el fin de proponer un modelo de desarrollo a través de un proceso cíclico de formación de identidad.

\footnotetext{
${ }^{4}$ El autor habla de la identidad cuando señala que el self se deriva de la relación con los otros, surgiendo de dicha relación el "mí", que se refiere al componente sociológico y al "yo" que se refiere al componente personal.
} 
En dicho proceso interactúan tres componentes: a) el aprendizaje conceptual (obtenido por lecturas, seminarios, artículos), b) el aprendizaje experiencial (aprendizaje que ocurre durante las clases aplicadas, internados y prácticas profesionales) y c) la evaluación externa (recibiendo información y retroalimentación sobre sus actuaciones en voz de pares, supervisores, profesores y clientes).

Este ciclo de aprendizaje conceptual, experiencial y de evaluación externa, apoya el desarrollo de la identidad del terapeuta en formación mediando la identificación, clarificación y re-clarificación de su auto-concepto.

Finalmente, Gibson, Dollarhide y Moss (2010) proponen un modelo de desarrollo integral, en el que incluyen los componentes intrapersonales e interpersonales para conceptualizar los progresos en la identidad profesional de los terapeutas.

En su propuesta holística, los autores definen la identidad profesional como "una integración exitosa de los atributos personales y del entrenamiento profesional en el contexto de una comunidad profesional" (Gibson et al., 2010, p. 23-24).

Los autores analizaron por medio de entrevistas semiestructuradas y grupos focales la experiencia profesional de 43 terapeutas en diferentes niveles de su formación.

Con base en dicha información, derivaron un modelo de desarrollo en el que ubican tres tareas que describen la identidad profesional, a saber: 1) definición internalizada de la terapia, 2) responsabilidad internalizada del crecimiento profesional, y 3) una identidad sistémicamente integrada con la comunidad profesional.

Este proceso involucra que, a través del trabajo, experiencia y compromiso, el terapeuta en formación pase de la validación externa (proporcionada por profesores, colegas y supervisores) hasta la autovalidación, que implica el compromiso con el aprendizaje continuo, un sentido de unión con la comunidad profesional y la integración de la identidad personal con la profesional.

En síntesis, los trabajos realizados bajo esta línea de investigación han aportado datos importantes respecto a las características y experiencias que impactan en la identidad profesional del psicólogo a través de los diferentes momentos de su formación en el área clínica. Sin embargo, desde estos trabajos, los componentes sociales que intervienen en la construcción de una identidad profesional aparecen desdibujados, ya que sólo se alude al contexto en el que el psicólogo desarrolla su actividad profesional y no se analizan elementos referentes a cómo esa identidad es construida y negociada dentro de una comunidad profesional.

La siguiente línea de trabajo se interesa particularmente por analizar cómo se construyen y negocian las identidades profesionales del psicólogo en vinculación con los contextos en los que participan.

\section{Construcción de la identidad del psicólogo}

Nyström (2008) y Nyström, Dahlgren y Dahlgren (2008) se han interesado por explorar la construcción de la identidad profesional como una relación entre lo profesional y los aspectos personales de vida. Para estos autores, la identidad profesional es un proceso de negociación, dinámico y vinculado a los contextos sociales de los que son participes los estudiantes en formación.

Con el fin de probar su tesis, los autores realizaron una investigación longitudinal en la que entrevistaron a estudiantes de dos programas formativos; once del programa de Psicología y ocho del programa de Ciencias Políticas y Economía, de una universidad sueca, en tres diferentes momentos de su formación, tanto dentro como fuera de las aulas: 1) en el último semestre antes de graduarse, 2) de 15 a 18 meses después de graduarse y al estar ya ejerciendo su profesión, y 3 ) cuando tenían de 30 a 34 meses de trabajar en un empleo relacionado con su área profesional.

Los hallazgos apuntalan que los graduados forman su identidad profesional a través de una conexión entre las diferentes esferas de su vida, la profesional, la privada y la personal. A su vez, en la transición de la educación superior a la vida laboral se producen tres diferentes formas identitarias (identidad no-diferenciada, segmentada e integrada) las cuales ejemplifican diferentes relaciones negociadas entre las esferas profesionales, personales $y$ privadas.

Dichas identidades pueden ser vistas como una secuencia evolutiva en los graduados; transitan de un foco individual a uno más relacional e integrado en formas de razonar acerca de la profesión. Es decir que la formación de la identidad profesional es una relación dinámica entre las diferentes esferas y no un fenómeno aislado que sólo toma lugar en la universidad o el contexto de trabajo.

En este tenor, la investigación de Tanggaard y Elmholdt 
(2007) analiza, a través del estudio de caso de dos psicólogos educativos en Dinamarca, las trayectorias de aprendizaje y la identidad profesional de éstos. Las trayectorias de aprendizaje son entendidas como el proceso mediante el cual los individuos combinan y conectan sus aprendizajes a través del tiempo y en diferentes espacios de trabajoy educación. Se realizaron 15 entrevistas semiestructuradas, que se relacionaban con la pregunta principal de la investigación: ¿cómo los psicólogos educativos responden a los cambios en las instituciones educativas y qué impacto tienen éstos en su identidad profesional?

Los resultados indican que, en los dos casos estudiados, el aprendizaje es percibido como una cuestión de descubrimiento y negociación de sus sentidos personales con la participación en las actividades institucionalizadas, métodos y tradiciones en el trabajo psicológico. En ambos casos se muestra la identidad profesional como una continua negociación de las dificultades de la práctica diaria y las propias expectativas de ser psicólogos en un contexto escolar.

Finalmente, el trabajo de Reid, Dahlgren, Petocz y Dahlgren (2008) aportan evidencias de que la identidad profesional está vinculada con el compromiso a la profesión, las concepciones sobre el conocimiento disciplinario y el trabajo profesional.

Realizaron un estudio longitudinal con 500 estudiantes, en diferentes momentos de su formación, y con recién graduados con experiencias en la práctica profesional. Los participantes de la investigación venían de tres campos disciplinarios en universidades de Australia y Suecia (Ciencias Políticas, Matemáticas y Psicología).

Por lo que respecta a los psicólogos, éstos reportaron ver el conocimiento como relativo, es decir, que tenían que sopesar y negociar los significados de los contenidos para llegar a una construcción del conocimiento dentro de su disciplina. Asimismo, al acercarse al ejercicio profesional, también presentaban formas relativistas de conocimiento cuando se enfrentaban a la incertidumbre de los problemas, y tenían que confrontar sus diferentes perspectivas para resolver los dilemas profesionales.

Los hallazgos de este equipo de investigadores indican que los psicólogos construyen su identidad en constante negociación con el grupo profesional y que el aprendizaje basado en problemas posibilita el desarrollo del compromiso con la disciplina y su identidad como profesionales de psicología.
De esta manera, a través de dicha línea de investigación se documenta que la construcción de la identidad profesional está estrechamente vinculada a los contextos sociales en los que el estudiante participa, y no se limita al contexto de la universidad o el trabajo. Las características de estos contextos pueden favorecer la negociación y re-negociación del rol profesional de los estudiantes en formación para que, eventualmente, desplieguen formas más activas de concebirse como profesionales legítimos en su práctica profesional.

Si bien estos antecedentes consolidan una valiosa aportación al estudio de la identidad profesional del psicólogo, los datos se circunscriben solamente a países como Estados Unidos de América y los países europeos de habla no hispana, por lo que existe un importante vacío en el estudio de la identidad profesional en Iberoamérica.

De ahí que a continuación nos proponemos indagar los elementos implicados en el proceso de construcción de la identidad profesional del psicólogo en las investigaciones reportadas en el contexto iberoamericano.

\section{Método}

\section{Rango de la búsqueda}

Para llevar a cabo el análisis sistemático de la literatura, se realizó un estudio de tipo cualitativo-interpretativo, el cual tiene por objetivo obtener una interpretación científico-humanista de la perspectiva teórica y los resultados de las investigaciones (Fernández-Ríos y Buela-Casal, 2009).

\section{Unidades de análisis}

El procedimiento de recopilación de los artículos se realizó a través de una búsqueda especializada en las principales bases de datos de Iberoamérica (Scielo, Redalyc, Dialnet y Doaj).

\section{Núcleos temáticos}

Asimismo, la búsqueda se realizó con base en cuatro series de descriptores que contuvieran las palabras clave que versaran sobre: 1) identidad profesional-Psicología, 2) psicólogos-identidad, 3) estudiantes de Psicologíaidentidad, y 4) construcción identidad-psicólogos.

\section{Artículos encontrados}

A través de dicha exploración, recuperamos un total de 
sesenta y tres artículos (18 de Redalyc, 17 de Scielo, 13 de Dialnet y 15 de Doaj).

\section{Composición de la muestra de artículos}

De este conjunto de artículos seleccionamos sólo cinco (3 de Redalyc, 1 de Scielo y 1 de Doaj), debido a que cincuenta y tres investigaciones centraban sus objetivos en temáticas diferentes a la que se buscaba analizar (la identidad profesional del psicólogo), tres investigaciones correspondían a reseñas de libros y dos a revisiones teórico-empíricas del concepto de identidad profesional.

\section{Criterio de inclusión y exclusión de artículos}

Los artículos fueron seleccionados mediante el cumplimiento de tres criterios de inclusión, a saber: 1) trabajo de investigación empírico, 2) estudiantes o graduados de la carrera de Psicología, y 3) publicadas durante el periodo 2010-2013.

\section{Procedimiento}

La información obtenida de los artículos seleccionados fue analizada a través de la identificación de indicadores contextuales y temáticos. Para el primero, se organizaron los datos de acuerdo a su orden cronológico y se identificaron los siguientes aspectos: a) año de publicación, b) autores, c) título del artículo, d) revista, e) país de publicación y, f) idioma. Para los segundos, se resaltaron los siguientes rubros: a) objetivo, b) participantes, c) método, y d) principales resultados.

\section{Resultados}

De los cinco artículos seleccionados, identificamos, a través de los indicadores contextuales, un mayor interés de investigación en el tema de identidad profesional en el último año (ver Tabla 1), esto se debe a que quizá es un campo de investigación aún en ciernes y de reciente conformación en Iberoamérica. Asimismo, el país en el que más se ha investigado sobre la identidad profesional del psicólogo es México, aunque cabe destacar que Brasil y Chile también han hecho aportaciones al tema.

Por otro lado, a través de los indicadores temáticos, observamos un predominio en el uso de enfoques cualitativos para el estudio de la identidad profesional, con un marcado uso de la entrevista, como herramienta, en sus diferentes modalidades (ver Tabla 2).

Con respecto a las características de los participantes, la mayoría de las investigaciones se llevaron a cabo con estudiantes de psicología de los últimos semestres de la carrera. Otra se realizó con estudiantes de maestría en psicología escolar y una más con psicólogos de recién inserción al campo laboral.

Al cuestionarnos sobre los elementos implicados en la construcción de la identidad profesional del psicólogo, dentro de la literatura analizada, identificamos cuatro dimensiones que abarcan cuestiones relativas a: 1) características de los contextos profesionales, 2) vínculo entre epistemología e identidad profesional,

Tabla 1.

Indicadores contextuales de las investigaciones revisadas sobre identidad profesional del psicólogo y en las modalidades evaluadas

\begin{tabular}{|c|c|c|c|c|c|c|}
\hline Año & Autor(es) & Título del artículo & Revista & País de publicación & Idioma & Base de datos \\
\hline 2010 & $\begin{array}{l}\text { Laport, Becker, } \\
\text { De Sarratea y } \\
\text { Ocampo }\end{array}$ & $\begin{array}{l}\text { Vinculación laboral flexible: } \\
\text { construcción de identidad } \\
\text { laboral en psicólogos adultos } \\
\text { jóvenes. }\end{array}$ & $\begin{array}{l}\text { Psicoperspectivas, } \\
\text { Individuo y } \\
\text { Sociedad }\end{array}$ & Chile & Español & Scielo \\
\hline 2011 & Díaz y Flores & $\begin{array}{l}\text { El cambio en el pensamiento } \\
\text { profesional del psicólogo escolar } \\
\text { en formación. }\end{array}$ & Perfiles Educativos & México & Español & Redalyc \\
\hline 2013 & Covarrubias & $\begin{array}{l}\text { Imagen social e identidad } \\
\text { profesional de la psicología } \\
\text { desde la perspectiva de sus } \\
\text { estudiantes. }\end{array}$ & $\begin{array}{l}\text { Revista } \\
\text { Iberoamericana de } \\
\text { Educación Superior }\end{array}$ & México & Español & Redalyc \\
\hline 2013 & $\begin{array}{l}\text { Alonso y Melo- } \\
\text { Silva }\end{array}$ & $\begin{array}{l}\text { Evaluación de una intervención } \\
\text { en orientación profesional en la } \\
\text { perspectiva de ex practicantes. }\end{array}$ & $\begin{array}{l}\text { Psicología: Ciência e } \\
\text { Proffissão }\end{array}$ & Brasil & Portugués & Doaj \\
\hline 2013 & Rodríguez y Seda & $\begin{array}{l}\text { El papel de la participación de } \\
\text { estudiantes de Psicología en } \\
\text { escenarios de práctica en el } \\
\text { desarrollo de su identidad } \\
\text { profesional. }\end{array}$ & Perfiles Educativos & México & Español & Redalyc \\
\hline
\end{tabular}


Tabla 2 .

Indicadores temáticos de las investigaciones revisadas sobre identidad profesional del psicólogo y en las modalidades evaluadas

\begin{tabular}{|c|c|c|c|c|}
\hline Autor (es) & Objetivo & Participantes & Instrumentos/Análisis & Principales resultados \\
\hline $\begin{array}{l}\text { Laport, Becker, De } \\
\text { Sarratea y Ocampo } \\
\text { (2010) }\end{array}$ & $\begin{array}{l}\text { Conocer y analizar las } \\
\text { construcciones de identidad laboral } \\
\text { que desarrollan profesionales entre } \\
25 \text { y } 34 \text { años, psicólogos en } \\
\text { condición de vinculación laboral } \\
\text { flexible. }\end{array}$ & $\begin{array}{l}9 \text { profesionales de la } \\
\text { Psicología }\end{array}$ & $\begin{array}{l}\text { Entrevista } \\
\text { semiestructurada/Análisis } \\
\text { de discurso. }\end{array}$ & $\begin{array}{l}\text { Cuando el contexto laboral se } \\
\text { presenta como inestable, la agencia } \\
\text { y la capacidad de control profesional } \\
\text { se reafirman. }\end{array}$ \\
\hline Díaz y Flores (2011) & $\begin{array}{l}\text { Analizar cómo el psicólogo en } \\
\text { formación logra desarrollar una } \\
\text { perspectiva profesional propia. }\end{array}$ & $\begin{array}{l}3 \text { psicólogos del } \\
\text { último semestre de la } \\
\text { maestría en } \\
\text { Psicología Escolar }\end{array}$ & $\begin{array}{l}\text { Entrevistas en formato } \\
\text { abierto guiadas por } \\
\text { observación de } \\
\text { videos/análisis con base en } \\
\text { el modelo de Perry (1981). }\end{array}$ & $\begin{array}{l}\text { El cambio a un pensamiento } \\
\text { relativista por parte del psicólogo } \\
\text { ocurre cuando éste integra el } \\
\text { conocimiento científico y el } \\
\text { experiencial, y reconoce la visión de } \\
\text { sus clientes y compañeros de } \\
\text { trabajo. }\end{array}$ \\
\hline Covarrubias (2013) & $\begin{array}{l}\text { Analizar las representaciones que } \\
\text { tienen estudiantes de Psicología } \\
\text { sobre la imagen social de la } \\
\text { Psicología y su identidad profesional. }\end{array}$ & $\begin{array}{l}22 \text { estudiantes del } \\
\text { último semestre y } \\
\text { algunos egresados de } \\
\text { Psicología }\end{array}$ & $\begin{array}{l}\text { Entrevista } \\
\text { semiestructurada/proceso } \\
\text { analítico-interpretativo. }\end{array}$ & $\begin{array}{l}\text { Si la formación profesional no } \\
\text { articula la teoría con la práctica, el } \\
\text { estudiante se percibe desorientado, } \\
\text { sin rumbo y con una identidad } \\
\text { profesional incierta, difusa e } \\
\text { imprecisa. }\end{array}$ \\
\hline $\begin{array}{l}\text { Alonso y Melo-Silva } \\
\text { (2013) }\end{array}$ & $\begin{array}{l}\text { Evaluar los procesos y resultados de } \\
\text { una intervención en orientación } \\
\text { profesional desde la percepción de } \\
\text { los egresados. }\end{array}$ & $\begin{array}{l}33 \text { estudiantes del } 9^{\circ} \\
\text { semestre de } \\
\text { Psicología }\end{array}$ & $\begin{array}{l}\text { Cuestionario de Evaluación } \\
\text { en Orientación } \\
\text { Profesional/análisis mixto. }\end{array}$ & $\begin{array}{l}\text { Establecer un vínculo con el cliente } \\
\text { es importante para hacer eficaz la } \\
\text { intervención y fortalecer la } \\
\text { identidad profesional del } \\
\text { practicante. }\end{array}$ \\
\hline $\begin{array}{l}\text { Rodríguez y Seda } \\
\text { (2013) }\end{array}$ & $\begin{array}{l}\text { Analizar lo que estudiantes de } \\
\text { Psicología significan sobre su } \\
\text { profesión a partir de su participación } \\
\text { en contextos de investigación e } \\
\text { intervención. }\end{array}$ & $\begin{array}{l}34 \text { estudiantes de la } \\
\text { carrera de Psicología } \\
\text { en diferentes } \\
\text { escenarios formativos }\end{array}$ & $\begin{array}{l}\text { Elaboración de } \\
\text { autoreportes/análisis de } \\
\text { protocolos verbales. }\end{array}$ & $\begin{array}{l}\text { La participación en escenarios } \\
\text { profesionales posibilita ejercer el rol } \\
\text { profesional, aprender } \\
\text { conjuntamente con colegas y } \\
\text { desarrollar autonomía y sentido y } \\
\text { pertenencia a un grupo de } \\
\text { profesionales. }\end{array}$ \\
\hline
\end{tabular}

3) formación profesional vinculada a la práctica, e 4) interacción psicólogo-cliente.

1) Características de los contextos profesionales: en su estudio sobre la relación entre identidad profesional y los contextos laborales flexibles Laport, Becker, De Sarratea y Ocampo (2010) exploraron, por medio de entrevistas semiestructuradas, las perspectivas de nueve profesionales de la Psicología que se encontraban trabajando en condiciones laborales flexibles, es decir, que no contaban con un contrato estable y definido. A través de sus narraciones, los profesionales reconstruyen sus identidades profesionales con base en la inestabilidad del contexto en el que laboran, el cual les demanda estar en constante movilidad y les imposibilita hacer proyectos profesionales a largo plazo. Las dificultades del contexto laboral aparecen en su discurso como una manera de reafirmar su agencia personal, la capacidad de control y la toma de decisiones profesionales.

2) Vínculo entre epistemología e identidad profesional: la investigación de Díaz y Flores (2011) arroja importantes resultados respecto a cómo los psicólogos en formación desarrollan una perspectiva profesional propia. En este estudio participaron tres psicólogos de una maestría en Psicología escolar que fueron entrevistados al inicio y al final de su último semestre de formación, con base en videograbaciones de su práctica profesional. Se documenta un cambio a formas relativistas de pensamiento, que responde a aspectos como el comprender de forma integral el conocimiento científico y experiencial, y reconocer la visión del cliente y de los compañeros de trabajo. Asimismo, dichas formas de pensamiento posibilitan el desarrollo de la identidad profesional y de un compromiso con la disciplina.

3) Formación profesional vinculada a la práctica: un elemento crucial para la construcción de la identidad profesional en estudiantes en formación es la participación en espacios de práctica profesional. Esto se ha documentado en dos investigaciones. En una de ellas, Covarrubias (2013) exploró las representaciones sociales de veintidós estudiantes de Psicología, del último semestre y graduados, que tenían sobre la imagen social su propia identidad profesional. En su análisis identificó que la mayoría de los estudiantes consideraban con indeterminación social e 
imprecisión profesional a la disciplina.

Dichas representaciones estaban vinculadas con las experiencias que tuvieron durante su formación profesional, en las que se privilegiaba el conocimiento teórico y se descuidaba el acercamiento al ejercicio profesional. Este tipo de formación contribuye a que el estudiante se sienta desorientado y con una identidad profesional incierta, difusa e imprecisa.

Lo anterior va de la mano con los hallazgos de Rodríguez y Seda (2013), en los que se documentan los significados que treintay cuatro estudiantes de Psicología construyen a través de su participación en diversos escenarios profesionales (laboratorios de investigación, centros de atención psicológica e instituciones educativas y de salud). Los estudiantes valoraron positivamente su experiencia en escenarios de práctica profesional, en particular, el haber ejercido las funciones propias del psicólogo, ser acompañados por un tutor académico y aprender conjuntamente con los colegas de trabajo, con los que compartía responsabilidades y ejercitaban la toma de decisiones y autonomía. Asimismo, estos estudiantes desarrollaron un sentido de pertenencia al grupo de profesionales.

4) Interacción psicólogo-cliente: en el trabajo realizado en Brasil por los investigadores Alonso y Melo-Silva (2013), se evaluó el proceso y resultados de las intervenciones que realizaron treinta y tres estudiantes de Psicología durante su participación en el servicio de orientación profesional en una universidad pública. Para recabar información aplicaron un cuestionario en el que se evaluaron diferentes aspectos del servicio, entre ellos la interacción psicólogocliente. En sus comentarios, los practicantes consideraron como positivas las estrategias de intervención que se manejaban en el servicio de orientación y señalaron la importancia que tiene para su identidad profesional el establecer un vínculo con los clientes, en el cual encuentran que la intervención se vuelve más eficaz si consideran la realidad contextual de los clientes, son espontáneos y se ajustan a nuevas experiencias que no siempre corresponden con los objetivos de la intervención.
Dichos resultados coinciden con las dimensiones identificadas en modelos recientes que analizan la interacción entre aprendizaje e identidad profesional (Reid, Abrandt Dahlgren, Petocz y Dahlgren, 2011). En dichos modelos se señala que la identidad profesional se desarrolla a través de la conexión entre las concepciones del conocimiento profesional y las actividades que se desarrollan en el contexto de trabajo o práctica profesional. Es en este vínculo que el estudiante en formación se convierte progresivamente en profesional, al compartir un compromiso tanto a nivel de la participación en su práctica, en el uso de técnicas, como a nivel ético, en el que articula sus valores personales y profesionales para atender a los dilemas de la práctica profesional.

En suma, los hallazgos de las investigaciones analizadas muestran indicios de que la construcción de la identidad profesional del psicólogo no es un asunto que se quede meramente en el conocimiento disciplinar y en las aulas; por el contrario, dichas investigaciones señalan que ésta se encuentra estrechamente vinculada al ejercicio profesional y a la participación activa del estudiante en la resolución de problemas en la práctica, lo cual no sólo posibilita cambios en las destrezas y habilidades del psicólogo, sino que, también, promueve formas de pensamiento que conducen hacia la autonomía y el compromiso profesional.

A través de este panorama hemos delineado algunos de los elementos implicados en la construcción de la identidad profesional del psicólogo, los cuales discutiremos a continuación con el fin de reflexionar sobre los hallazgos hasta ahora documentados.

\section{Discusión}

La revisión de las investigaciones presentadas apuntalan algunos de los elementos integrantes del proceso de construcción de la identidad profesional, a saber: la manera en que el estudiante concibe su propio conocimiento profesional (Rønnestad y Skovholt, 2003; Gibson et al., 2010; Díaz y Flores, 2011) y la formación profesional vinculada a la práctica (Nyström, 2008; Nyström et al., 2008; Reid et al., 2008; Covarrubias, 2013; Rodríguez y Seda, 2013). En esta línea, nos parece que el análisis de ambas cuestiones constituye el punto de articulación entre las posturas que aparecen como dislocadas en el estudio de la identidad profesional.

Lo anterior puede ser comprendido a la luz de los estudios sobre epistemología personal, los cuales abarcan diferentes investigaciones sobre las teorías o creencias que las personas construyen acerca del 
conocimiento y el proceso de conocer (Hofer, 2001).

Afín con la idea de que el desarrollo del conocimiento implica, a su vez, un cambio en la forma de ver el mundo y de posicionarse ante éste, en diversos estudios se ha resaltado el vínculo entre la identidad y la epistemología personal. Desde el trabajo pionero de Perry (1970) se señaló la identidad como un importante elemento en el desarrollo personal, puesto que contribuye al cambio epistemológico que se da en el tránsito de formas dualistas a formas relativistas de pensamiento, esto es, que la persona pasa de tener una visión simple e incuestionable del conocimiento a una posición en el que reconoce al conocimiento como construido, complejo, tentativo y circunscrito a la evidencia.

Trabajos más recientes han evidenciado el vínculo entre identidad y aprendizaje (Baxter Magolda, 2003, 2004; King y Baxter Magolda, 2005) al ser la identidad un elemento central para promover el aprendizaje, que la persona construya un sentido de sí misma que guíe sus decisiones personales y profesionales, y para que evolucione hacia formas de conocimiento personal más complejas en las que la auto-autoría (selfauthorship) aparece como la capacidad de las personas para asumir un papel activo en sus decisiones y dar sentido a sus creencias, a su conocimiento y a la construcción de su self. Al respecto, se ha discutido sobre la auto-autoría como un constructo que permite acercarse holísticamente a la construcción de una identidad reflexiva del Yo (Bontempo, Flores y Ramírez, 2012).

Partiendo de estos postulados, es posible considerar que las creencias que desarrollan las personas acerca del conocimiento y del mundo se relacionan estrechamente con la construcción de sus identidades, al ser dichas creencias las que le proporcionan un marco de referencia con el que construyen una noción de sí mismas, de su ética y de su papel en la relación con los otros. Así, en el proceso de construcción de la identidad profesional, las creencias acerca del conocimiento disciplinario aparecen como un importante elemento que le permiten al profesionista llegar a formas complejas de razonamiento intelectual y ético.

En el caso de los psicólogos en formación, se ha documentado que éstos, al llegar a formas relativistas de concebir su conocimiento en torno a la profesión, logran lidiar con la incertidumbre de los problemas profesionales, construyen una postura propia sobre su papel como profesionistas, desde la cual reflexionan sobre sus experiencias y sobre sí mismos como profesionales, además de que el conocimiento que deriva de dicha reflexión les permite comprenderse en relación con los otros y, por ende, considerar e integrar las perspectivas de los usuarios de sus servicios, sus compañeros de trabajo y demás integrantes de una comunidad profesional.

Para que este proceso tenga lugar, se plantea la importancia de un contexto de formación en la práctica profesional, en el que es central la interacción del estudiante con sus pares y con expertos en diferentes dominios, para dar sentido a la variedad de dilemas profesionales a los que se enfrenta (Flores, Otero y Lavallée, 2010).

De esta manera, el cambio epistemológico que implica llegar a formas de pensamiento relativistas sobre el conocimiento profesional, permite que el estudiante tenga elementos para construir una noción de sí mismo como profesionista y asuma un compromiso ético con su profesión; que transite de una visión de su actuación profesional orientada por fuentes externas y supeditada a las evaluaciones de las figuras de autoridad que participan en su proceso de formación, a una visión internalizada, en la que se autoevalúa, reflexiona sobre su propia actuación y considera las perspectivas de los otros en su toma de decisiones.

Por otra parte, la manera en la que el conocimiento profesional es conceptualizado por los estudiantes se encuentra estrechamente vinculado a la membrecía que mantienen con la comunidad profesional, aspecto que también ha sido descrito como fundamental en la construcción de la identidad profesional (Nyström et al., 2008; Nyström, 2008; Covarrubias, 2013; Rodríguez y Seda, 2013).

De acuerdo con esto, los cambios por los que transita el estudiante en su formación profesional se movilizan por medio de dos procesos: el desarrollo personal y la membrecía a una comunidad profesional. Esta idea coincide con lo que Wenger (1998) señala cuando habla de que "la membrecía con una comunidad de práctica se traduce en una identidad como una forma de competencia" (pg. 153).

Es así que el desarrollo de las habilidades del estudiante no puede ser separado de los procesos sociales en los que tienen lugar las experiencias profesionales y las diversas situaciones a las que éste se va enfrentando para dar resolución a los problemas de su profesión. Del mismo modo, dichos problemas son indispensables para fomentar en él un progreso, puesto que son el 
escenario perfecto para que evalúe de manera integral sus conocimientos teóricos y prácticos, y reflexione con base en la evidencia sobre su postura y compromiso ético (Hofer, 2004).

En síntesis, considerando los aportes de ambas perspectivas, podemos proponer que para aproximarse al estudio de la identidad profesional es necesario realizar análisis integrales, en los que se consideren los cambios epistemológicos y las características de los contextos profesionales en los que participan los estudiantes en formación. De esta manera, las brechas de investigación actuales encontrarán puntos de intersección que posibiliten la comprensión de los procesos imbricados en la construcción de la identidad profesional.

\section{Conclusiones}

A la luz de la literatura analizada nos propusimos reflexionar en torno a los elementos implicados en la construcción de la identidad profesional del psicólogo. En el campo de la identidad profesional existen dos brechas de investigación, al ser ésta un constructo que se encuentra en una suerte de dislocamiento entre un polo personal y un polo social, lo que dificulta que se pueda llegar a una comprensión integral de su estudio (Trede, Macklin y Bridges, 2012; Ruvalcaba-Coyaso, Uribe y Gutiérrez, 2011). En ello radica la importancia de reflexionar sobre los elementos que posibilitan su desarrollo.

Con base en los hallazgos de distintas investigaciones, podemos deducir que uno de los elementos que modula el proceso de construcción de la identidad profesional son las creencias epistemológicas que el estudiante construye en torno a su conocimiento profesional y a su rol en la profesión. Asimismo, el cambio epistemológico de formas dualistas a formas relativistas de pensamiento está estrechamente vinculado con el papel de las experiencias profesionales y la participación activa de los estudiantes en la comunidad profesional.

De esta manera, la identidad profesional se construye a través de un proceso dinámico, personal y social (Dubar, 1992) en el que el estudiante aprende a ser un cierto tipo de persona, que es reconocida por desplegar las características propias de un profesionista. En el proceso, el estudiante inicia su formación con un limitado conocimiento acerca de la profesión y una concepción desintegrada de lo que ésta implica. A la par de lo anterior, sus creencias acerca del conocimiento profesional se basan y dependen en gran medida de las fuentes de autoridad (libros, profesores, expertos).

Al principio de su formación, el profesionista es situado por los expertos y la comunidad como inexperto, lo que implica que sólo puede moverse en la periferia social de la profesión, y únicamente después de obtener cierto reconocimiento le es permitido participar en prácticas cada vez más complejas, que le posibiliten un papel más autónomo. Esto le permite construir formas de pensamiento más complejas y sofisticadas que le ayudan a resolver los problemas propios de la disciplina y a construir un sentido de pertenencia a la misma.

Al considerar el papel de la epistemología personal y los contextos profesionales en el análisis de la construcción de la identidad profesional, es posible entender los cambios por los que transita el estudiante en formación para construirse una imagen de sí mismo como profesional. Consideramos que la comprensión de dichos cambios llevará a replantear los programas formativos y las situaciones de enseñanza-aprendizaje que se manejan en las instituciones, propiciando una mayor comprensión de las situaciones y didácticas que favorecen en los estudiantes cambios epistemológicos hacia formas relativistas de conocimiento.

\section{Implicaciones para futuras investigaciones}

Derivado de lo analizado en el presente artículo, es plausible reconocer la importancia de realizar análisis integrales en el estudio de la identidad profesional. Si bien las investigaciones analizadas no abarcan en su totalidad el campo de estudio sobre la construcción de la identidad profesional del psicólogo, tampoco alcanzan a dar cuenta de las particularidades de dicho proceso en cada área de especialización de la disciplina psicológica (v.g. psicología clínica, educativa, social, laboral, de la salud), ni en cada una de las etapas formativas (pre-grado, maestría, doctorado), empero, nos muestran la importancia de realizar análisis integrales que unan las brechas de investigación respecto a cómo conceptualizar y caracterizar el proceso de construcción de la identidad profesional.

En este sentido, otra veta importante en el estudio de la identidad profesional del psicólogo es cómo ésta es construida en las diferentes realidades y programas formativos. Así, encontramos que gran parte de las investigaciones se han producido fuera del contexto iberoamericano, quedando pendientes por responder diversas interrogantes en nuestro contexto, a saber: qué papel juegan las instituciones de educación superior (IES) en la construcción de un 
sentido de pertenencia a la profesión, qué elementos de la formación recibida posibilitan que el estudiante se visualice como profesional, qué recursos apoyan el tránsito que los estudiantes realizan de las aulas universitarias a la práctica profesional.

Las futuras investigaciones en el área tendrán que ser capaces de responder las interrogantes planteadas y elaborar aproximaciones más integrales para analizar el proceso de construcción de la identidad profesional, las cuales, eventualmente, deriven en estrategias educativas que promuevan y faciliten que el estudiante construya una noción de sí mismo como profesional.

\section{Referencias}

Alonso, W.M. \& Melo-Silva, L. L. (2013). Evaluación de una intervención en orientación profesional en la perspectiva de ex practicantes. Psicologia: Ciência E Profissão, 33(1), 84-99.

Auxier, C.R., Hughes, F.R. \& Kline, W. B. (2003). Identity development in counselors in training. Counselor Education \& Supervision, 43, 25-43.

Baxter Magolda, M. B. (2003). Identity and Learning: Student Affairs' Role in Transforming Higher Education. Journal of College Student Development, 44(2), 231-247. doi:10.1353/ csd.2003.0020

Baxter Magolda, M. B. (2004). Evolution of a constructivist conceptualization of epistemological reflection. Educational Psychologist, 39(1), 31-42.

Beijaard, D., Meijer, P. C. \& Verloop, N. (2004). Reconsidering research on teachers' professional identity. Teaching and Teacher Education, 20(2), 107-128. doi:10.1016/j.tate.2003.07.001

Bontempo, L., Flores, R., Ramírez, L. (2012). La construcción de la identidad personal y el desarrollo de la auto-autoría. El Ágora USB, 12(2), 214-547.

Covarrubias, P. (2013). Imagen social e identidad profesional de la psicología desde la perspectiva de sus estudiantes. Revista Iberoamericana de Educación Superior, 4(10), 113-133.

Díaz, A. \& Flores, R. C. (2011). El cambio en el pensamiento profesional del psicólogo escolar en formación. Perfiles Educativos, 33(134), 6576.

Dollarhide, C. T., Gibson, D. M. \& Moss, J. M. (2013). Professional identity development of counselor education doctoral students. Counselor Education \& Supervision, 52(2), 137-150. doi:10.1002/j.1556-6978.2013.00034.x

Dubar, C. (1992). Formes identitaires et socialisation professionnelle. Revue Française de Sociologie, 33(4), 505-529. doi:10.2307/3322224

Ducheny, K., Alletzhauser, H. L., Crandell, D. \& Schneider, T. R. (1997). Graduate student professional development. Professional Psychology: Research and Practice, 28(1), 87-91. doi:10.1037//07357028.28.1.87

Elman, N. S., Illfelder-Kaye, J. \& Robiner, W. N. (2005). Professional development: Training for professionalism as a foundation for competent practice in psychology. Professional Psychology: Research and Practice, 36(4), 367-375. doi:10.1037/0735-7028.36.4.367

Fernández-Ríos, L. \& Buela-Casal, G. (2009). Standards for the preparation and writing of psychology review articles. International Journal of Clinical and Health Psychology, 9(2), 329-344.

Flores, C., Otero, A. \& Lavallée, M. (2010). La evolución de la perspectiva epistemológica en estudiantes de posgrado. Perfiles Educativos, 32(130), 8-24.

Gibson, D. M., Dollarhide, C. T. \& Moss, J. M. (2010). Professional identity development: A grounded theory of transformational tasks of new counselors. Counselor Education \& Supervision, 50(September), 21-38.

Goffman, E. (1959). Presentation of self in everyday life. New York: Doubleday Anchor Books.

Goldie, J. (2012). The formation of professional identity in medical students: considerations for educators. Medical Teacher, 34(9), e641-e648. doi:10.3109/0142159X.2012.687476

Hofer, B. K. (2001). Personal epistemology research : Implications for learning and teaching, 13(4), 353-383.

Hofer, B. K. (2004). Exploring the dimensions of personal epistemology in differing classroom contexts: Student interpretations during the first year of college. Contemporary Educational Psychology, 29, 129-163. doi:10.1016/j. cedpsych.2004.01.002

Johnson, M., Cowin, L. S., Wilson, I. \& Young, H. (2012). Professional identity and nursing: Contemporary theoretical developments and future research challenges. International Nursing Review, 59(4), 562-569. doi:10.1111/j.1466-7657.2012.01013.x

Kaslow, N. J. (2004). Competencies in professional psychology. American Psychologist, 59(8), 774781. doi:10.1037/0003-066X.59.8.774 
Kaufmann, J. C. (2004). L'invention de soi. Une théorie de l'identité. France: Hachette Littératures.

King, P. M. \& Baxter Magolda, M. B. (2005). A developmental model of intercultural maturity. Journal of College Student Development, 46(6), 571-592. Retrieved from http://muse. jhu.edu/journals/journal_of_college_student_ development/v046/46.6king.html

Laport, N., Becker, A., De Sarratea, A. \& Ocampo, G. (2010). Vinculación laboral flexible: Construcción de identidad laboral en psicólogos adultos jóvenes. Psicoperspectivas. Individuo Y Sociedad, 9(1), 138-157.

Nelson, K. \& Jackson, S. (2003). Professional counselor identity development: A qualitative study of hispanic student interns. Counselor Education \& Supervision, 43, 2-14.

Nyström, S. (2008). The dynamics of professional identity formation: Graduates' transitions from higher education to working life. Vocations and Learning, 2(1), 1-18. doi:10.1007/s12186-0089014-1

Nyström, S., Dahlgren, M. A. \& Dahlgren, L. O. (2008). A winding road - professional trajectories from higher education to working life: A case study of political science and psychology graduates. Studies in Continuing Education, 30(3), 215-229. doi:10.1080/01580370802439896

Perry, W. (1970). Forms of intellectual and ethical development in the college years. New York, N.Y.: Holt, Rinehart and Winston.

Reid, A., Dahlgren, L. O., Petocz, P. \& Dahlgren, M. A. (2008). Identity and engagement for professional formation. Studies in Higher Education, 33(6), 729-742. doi:10.1080/03075070802457108

Reid, A. Abrandt Dahlgren, M. Petocz, P. Dahlgren, L. (2011). Professional identity: How is professional identity developed? In Professional learning and development in schools and higher education (Vol. 5, pp. 85-100). New York, N.Y.: Springer. doi:10.1007/978-94-007-0250-9

Rodolfa, E., Bent, R., Eisman, E., Nelson, P., Rehm, L. \& Ritchie, P. (2005). A cube model for competency development: Implications for psychology educators and regulators. Professional Psychology: Research and Practice, 36(4), 347354. doi:10.1037/0735-7028.36.4.347 participación de estudiantes de Psicología en escenarios de práctica en el desarrollo de su identidad profesional. Perfiles Educativos, 35(140), 82-99.

Rønnestad, M. \& Skovholt, T. M. (2003). The journey of the counselor and therapist: Research findings and perspectives on professional development. Journal of Career Developmenter, 30(1), 5-44.

Ruvalcaba-Coyaso, J., Uribe, A.I. \& Gutiérrez, G. R. (2011). Identidad e identidad profesional: Acercamiento conceptual e investigación contemporánea. Revista CES Psicología, 4(2), 82-102.

Serra, M. N. (2008). Learning to be a nurse. Professional identity in nursing students. Sísifo. Educational Sciences Journal, 5, 65-76.

Tanggaard, L., \& Elmholdt, C. (2007). Learning trajectories among educational psychologists. Studies in Continuing Education, 29(3), 295-314. doi:10.1080/01580370701628508

Trede, F., Macklin, R. \& Bridges, D. (2012). Professional identity development: A review of the higher education literature. Studies in Higher Education, 37(3), 365-384. doi:10.1080/03075079.2010.521 237

UNESCO. (2009). Comunicado de la Conferencia Mundial sobre la Educación Superior 2009: la nueva dinámica de la educación superior y la investigación para el cambio social y el desarrollo. París: UNESCO. Retomado de: www.iesalc.unesco.org.ve/dmdocuments/ comunicado_cmes09es.pdf

Wenger, E. (1998). Communities of practice: Learning, meaning and identity. Cambridge: Cambridge University Press. 4.0 Internacional, por lo que su contenido gráfico y escrito se puede compartir, copiar y redistribuir total o parcialmente sin necesidad de permiso expreso de sus autoras con la única condición de que no se puede usar con fines directamente comerciales y los términos legales de cualquier trabajo derivado deben ser los mismos que se expresan en la presente declaración. La única condición es que se cite la fuente con referencia a la Revista Digital Internaciona de Psicología y Ciencia Social y a sus autoras. 


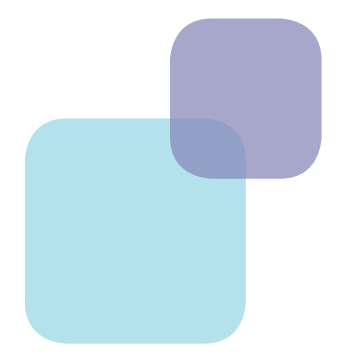

\section{MetA-ANÁLISIS DEL ARTículo}

A continuación se muestran los análisis de las puntuaciones otorgadas a este artículo por sus revisores
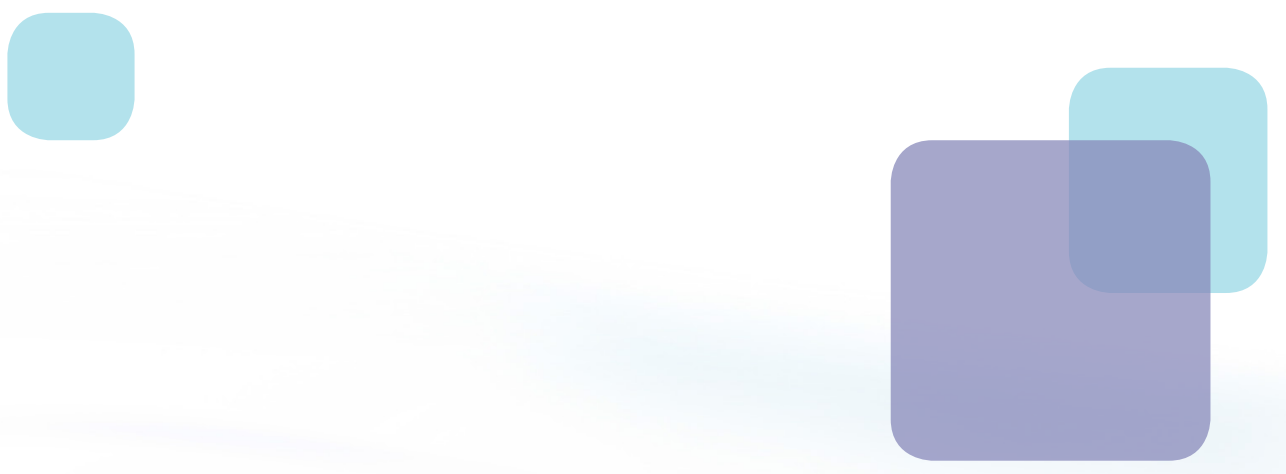


\section{Dimensión Cuantitativa}

\section{Perfil de Evaluación entre Pares}
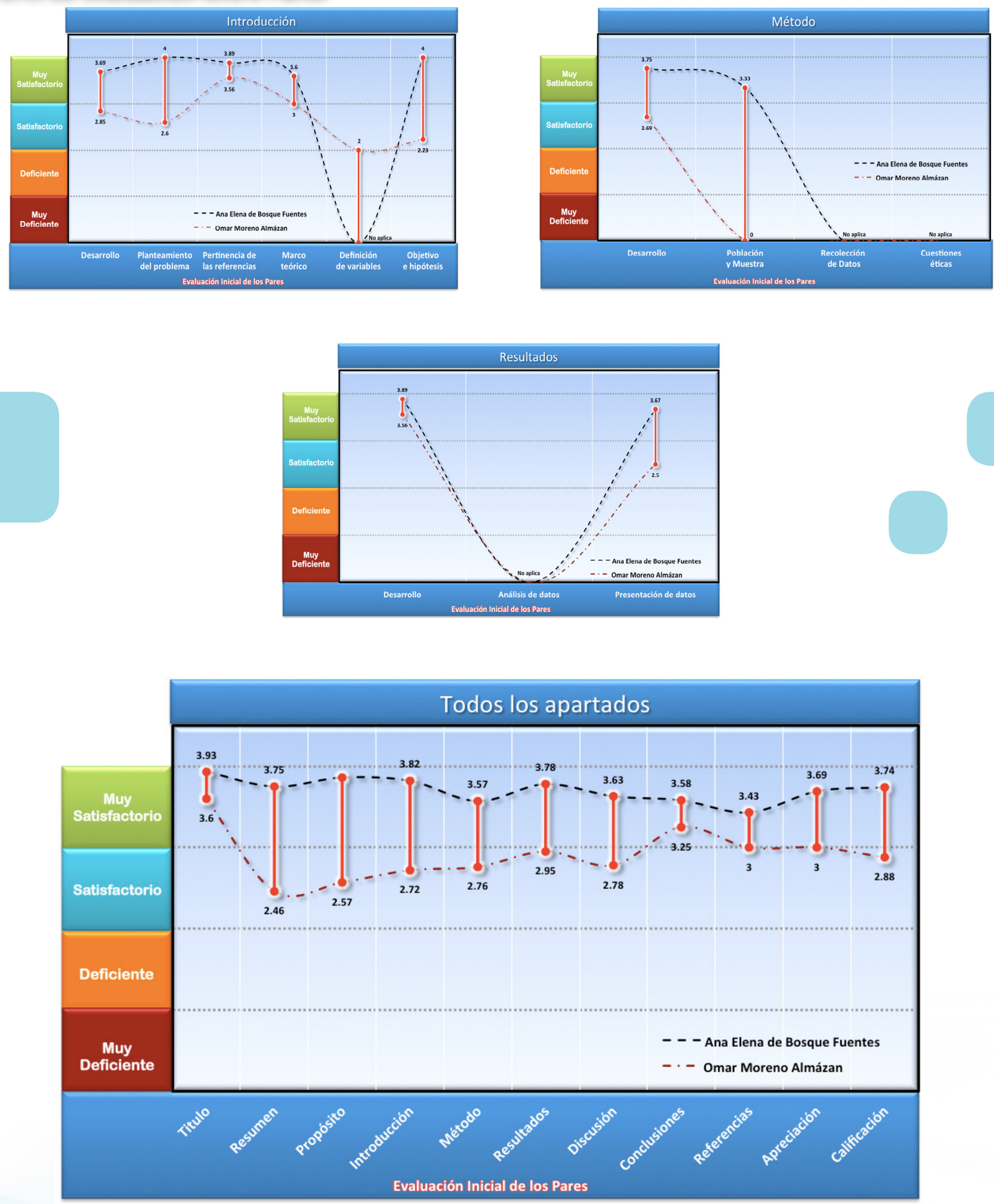


\section{Índice de Concordancia}

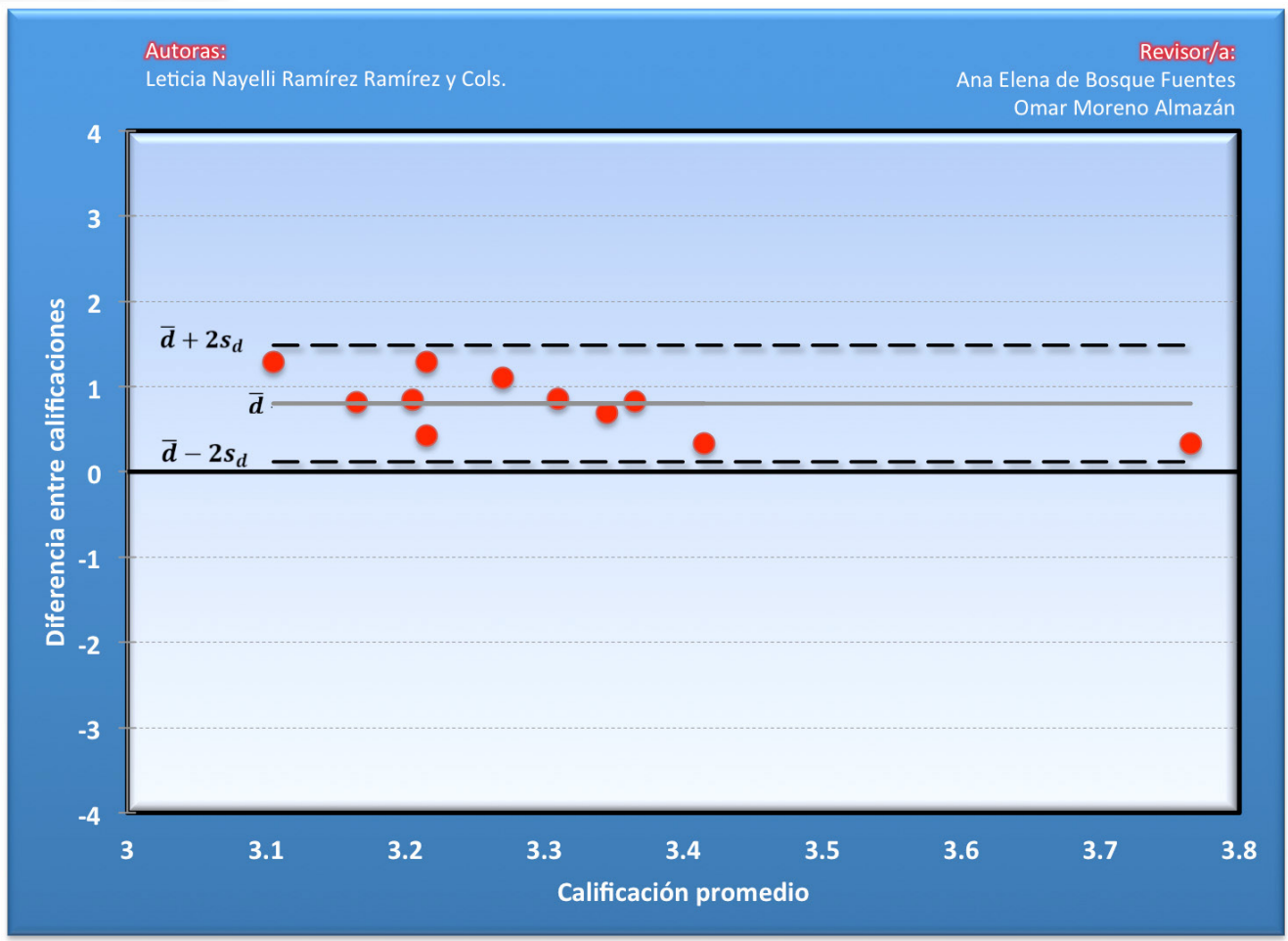

\section{Índice de Acuerdo}

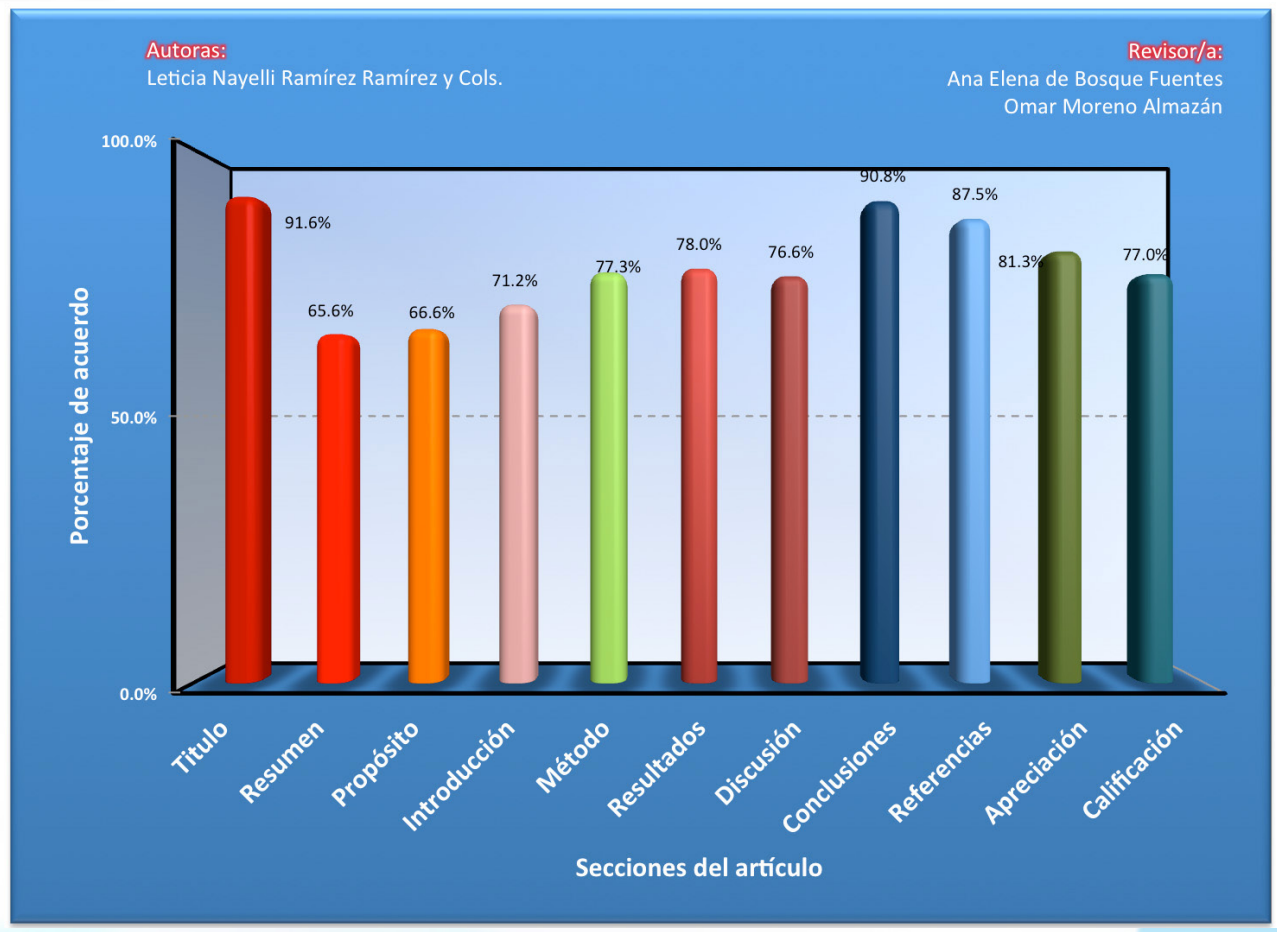




\section{Dimensión Cualitativa}

\begin{tabular}{|c|c|}
\hline REVISOR 1 & ReVISOR 2 \\
\hline Ana Elena del Bosque Fuentes & Omar Moreno Almazan \\
\hline \multicolumn{2}{|c|}{ TítuLo/AutORÍA } \\
\hline $\begin{array}{l}\text { El título del manuscrito es claro y explicativo del tema } \\
\text { que están abordando. La revisión de cinco artículos } \\
\text { elegidos para obtener los datos y el análisis que } \\
\text { hacen del tema no justifica de manera satisfactoria la } \\
\text { participación de cuatro autoras. Sólo se recomienda } \\
\text { que en futuros manuscritos se realice una mayor } \\
\text { profundidad del tema a tratar cuando la participación } \\
\text { implica a más de tres autores. }\end{array}$ & $\begin{array}{l}\text { El título en sí es bueno, solo que al leer el artículo } \\
\text { podríamos remitirnos en el imaginario, a un estudio de } \\
\text { meta-análisis muy extenso, lo cual no es así. }\end{array}$ \\
\hline \multicolumn{2}{|c|}{ RESUMEN } \\
\hline $\begin{array}{l}\text { El resumen es fácil de entender. Aun cuando contiene } \\
198 \text { palabras y se redacta en un solo párrafo, los } \\
\text { componentes se especifican, no necesariamente en } \\
\text { párrafos como se menciona en el formato pero estos } \\
\text { son claros y concisos. }\end{array}$ & $\begin{array}{l}\text { El resumen establece los objetivos pero no da una idea } \\
\text { completa del trabajo; de igual manera se espera un } \\
\text { meta-análisis más amplio. La explicación del análisis de } \\
\text { resultados es clara. }\end{array}$ \\
\hline
\end{tabular}

\section{Próposito del Estudio}

El tema es interesante, sin embargo, la forma en que lo abordan refiere más un estado del arte parcial al considerar solamente cinco artículos de acuerdo a los criterios que las autoras establecieron. El propósito del trabajo es redactado de manera clara, sin que necesariamente sea a partir de una pregunta de investigación.
La estructura que se maneja en el marco referencial y el método son relativamente congruentes; la sección de resultados es la que pierde el orden pues expone datos que son claros pero no con el apego que se señaló en las secciones anteriores.

\section{INTRODUCCIÓN}

Aunque el problema es relevante y actual, no implica de una medición, pero si de un análisis que podría ser más profundo, además de que sólo se consideraron cinco artículos para ser analizados. Si bien en el marco teórico se clarifican conceptos y sus relaciones, falta profundizar en algunos de ellos que son retomados posteriormente en las conclusiones y la discusión, caso concreto de la epistemología personal. No aplica el rubro de Variables de estudio, ya que es un trabajo teórico; a pesar de esto, se mencionan indicadores que guiaron el análisis de los datos. Considerando que es un trabajo de revisión de literatura, los rubros del método se presentan por cuestiones formales, sin ser necesaria esta manera de presentación, por lo tanto, se presenta el propósito del trabajo, que puede tomarse como el objetivo, no hay variables y tampoco formulación de hipótesis.
Da a entender una lógica en su idea principal, incluso los objetivos son ambiciosos; sin embargo a pesar de esa claridad no se aterriza en el artículo la claridad del tema. 


\section{Método}

La forma en que se presenta el método, aun cuando es clara, no parece apropiado para el propósito del estudio, que se centra más en una indagación teórica para conformar el estado del arte de un tema específico, por lo que la asignación de puntuación en esta sección habrá que tomarse con reservas.
Convendría que desde el marco teórico exista una definición de las dimensiones que se han tomado en cuenta, pues éstas son propuestas pero no se alcanzan a definir y no se ve reflejado en los resultados.

\section{Resultados}

Los resultados que se presentan son descriptivos y consideran los ejes de análisis propuestos; son claros aun cuando haya algunos detalles de redacción, además de que podría explicarse más la tabla dos.
Seguir la descripción de los datos tal y como se había planteado desde el principio, llevando el orden entre las dimensiones propuestas.

\section{Discusión}

La discusión presenta argumentos que se respaldan en investigaciones de otros autores, considera además los conceptos y las líneas de discusión establecidas, no obstante, no se profundiza en ellos.
La discusión a acorde con los resultados, al parecer de existir un control en el manejo de las dimensiones planteadas, podría tener un panorama relevante de lo que se ha estudiado. En ocasiones se hace comparación con los estudios previos, aunque esto es mínimo.

\section{CONCLUSIONES}

ILas conclusiones son claras y refieren los resultados del análisis teórico de los artículos revisados, sin embargo, considerando que sólo fueron cinco artículos, no se favorece para enfatizar y profundizar más sobre los aspectos a indagar, así como tampoco se hace un análisis crítico del estudio ni se proponen mejoras a futuro.
Un punto acertado en este artículo corresponde al contraste que se hace de los datos con lo que se halla en estudios similares; existe una idea de que se puede mejorar y se proponen líneas de investigación aún más específicas.

\section{REFERENCIAS}

Se reportan 40 referencias, que para la profundidad del análisis que hacen las autoras son excesivas, y en algunas solamente señalan aportaciones mínimas. En el texto no aparece la referencia de: Dollarhide, C. T., Gibson, D. M., \& Moss, J. M. (2013). Professional identity development of counselor education doctoral students. Counselor Education \& Supervision, 52(2), 137-150. doi:10.1002/j.1556-6978.2013.00034.x.

\section{IMPRESión General del Manuscrito}

En términos generales el trabajo es presentado de manera apropiada, salvo algunos mínimos detalles que se marcan en el texto.
Este apartado es adecuado.
Es un buen texto, aporta una idea actual de la noción de identidad de la psicología, sin duda podría estar más sólida en cuanto a sus referentes teóricos y la magnitud de la información adquirida, pero es un buen artículo.

\section{COMENTARIO POR DICTAMEN}

Al parecer la intención de la revista se centra en el desarrollo de temas relacionados con la psicología y el ámbito social. El artículo nos muestra una idea del desarrollo de la identidad de nuestra profesión a partir de la vinculación con lo social, con su desarrollo y la formación que se genera. Por ende el tema del artículo es sustancial. Podría mejorar su contenido a fin de darle mayor relevancia, sin duda eso ayudaría mucho más. 


\section{IR A LA HISTORIA DEL PROCESO EDITORIAL}


